\title{
IN VIVO WE TRUST
}

\section{The pharma- ceutical industry desperately needs the traditional skills of the pharmacologist}

Simon Frantz, Associate Editor (News), Nature Reviews Drug Discovery. e-mail:s.frantz@nature.com
During the past two decades, in vivo pharmacology suffered a downturn of interest in both industry and graduate recruitment. Once the molecular biology revolution convinced us that it would solve all of our problems, academics feared that their papers would not be published if they did not contain a whiff of molecular biology, and they amended courses to ensure that their students would be properly prepared for the brave new world. Students were attracted by the glamour of molecular biology, preferring to isolate a gene involved in cancer than to isolate the ileum of a guinea-pig. This, and increasing pressure from animal-rights movements, persuaded many universities that the financial and ethical costs of running large animal houses was not justified.

In the intervening years, molecular biology has indeed had a marked effect on drug discovery. With the availability of the human genome sequence data, an incredible number of potential targets for small-molecule drugs are being identified. However, in the enthusiasm to jump on the genomics bandwagon, unfashionable areas in the pharmaceutical industry such as in vivo pharmacology suffered as a result of their second-class status.

But, more than ever, the pharmaceutical industry is realizing that no matter what the origin of the compound you are testing, or how it came into being, a good description of its pharmacological properties is necessary to assess its drug-like potential. There have been major advances in the use of cell culture and recombinant human cells, and in silico approaches are also providing valuable alternatives to animal experiments by simulating drug interaction and response data. But, these studies still cannot predict the integrated response of a potential drug as accurately as living systems, in which a combination of genetic, biochemical, physiological, pathological and environmental influences work in concert. With regulatory bodies demanding more and more information about drug safety, companies need reliable data from animal studies on the therapeutic potential of compounds.

So, the technologies that threatened to make in vivo pharmacology extinct have, in fact, re-emphasized its importance in drug discovery. And while the industry needs in vivo pharmacologists more than ever to assess the targets being generated, the decreased number of students with the appropriate skills emerging from the academic system is a big worry. This is a particular problem in the United States, which faces a major skills shortage because of the low recruitment of pharmacologists by universities 10-20 years ago. The lack of experience in the area is, in some cases, causing recruiters to stretch the definition of in vivo pharmacologists to anyone who can find their way around a rat.

To address these issues, pharmacology societies and industry are joining forces to create appropriately trained in vivo pharmacologists. One of the biggest initiatives has been launched in the United Kingdom; the British Pharmacological Society (BOX 1) has set up funding with all the major UK pharmaceutical companies for around ten universities to run in vivo pharmacology modules. Students take time out (usually in their third year) and are given experience in the skills required for in vivo work. In addition, UK pharmaceutical companies also fund students to go on 'sandwich' courses. In the United States, Merck are collaborating with the American Society for Pharmacology and Experimental Therapeutics (ASPET; see BOX 1) to award postdoctoral fellowships in integrative pharmacology (the guidelines of which will be posted on ASPET's website soon).

Sir John Gaddum wrote ${ }^{1}$ almost 50 years ago that a pharmacologist can be described as a "jack of all trades", borrowing and building from all disciplines. His message is as relevant today as it was then - the type of skills that industry is looking for are a thorough grounding in physiology, physical chemistry, biology (including molecular biology) and therapeutics followed by high-level training/understanding of drug distribution and action. Developing skills and techniques in this field will be invaluable in helping companies to translate the glut of data spewing out from new technologies into new drugs.

Simon Frantz

Gaddum, J. H. The science of pharmacology. Nature 173, 14-15 (1954).

\section{Box 1 | Online resources for in vivo pharmacology}

The American Society for Pharmacology and Experimental Therapeutics (ASPET) ............http://www.aspet.org/ ASPET is an American scientific society that represents pharmacologists worldwide. It promotes research funding, publishes The Journal of Pharmacology and Experimental Therapeutics, Molecular Pharmacology, Pharmacological Reviews and Drug Metabolism and Disposition, and has a careers centre.

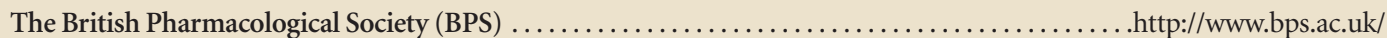
The BPS covers the whole spectrum of pharmacology, including in vivo research. It runs scientific meetings, publishes the British Journal of Pharmacology and the British Journal of Clinical Pharmacology, and provides careers materials.

General list of pharmacology societies .http://www.kfunigraz.ac.at/ekpwww/linksocl.html

Test your knowledge of pharmacology .................http://www.umu.se/pharm-neuro/inst/quiz/quiztop.html A fun guide to pharmacology from the University of Umeå, Sweden. 